\title{
Unbounded Positive Solutions and Mann Iterative Schemes of a Second-Order Nonlinear Neutral Delay Difference Equation
}

\author{
Zeqing Liu, ${ }^{1}$ Xiaochuan Hou, ${ }^{2}$ Jeong Sheok Ume, ${ }^{3}$ and Shin Min Kang ${ }^{4}$ \\ ${ }^{1}$ Department of Mathematics, Liaoning Normal University, Dalian, Liaoning 116029, China \\ ${ }^{2}$ Fushun No. 10 High School, Fushun, Liaoning 113004, China \\ ${ }^{3}$ Department of Mathematics, Changwon National University, Changwon 641-773, Republic of Korea \\ ${ }^{4}$ Department of Mathematics and RINS, Gyeongsang National University, Jinju 660-701, Republic of Korea
}

Correspondence should be addressed to Jeong Sheok Ume; jsume@changwon.ac.kr

Received 12 May 2012; Accepted 12 December 2012

Academic Editor: Alexander I. Domoshnitsky

Copyright (C) 2013 Zeqing Liu et al. This is an open access article distributed under the Creative Commons Attribution License, which permits unrestricted use, distribution, and reproduction in any medium, provided the original work is properly cited.

This paper is concerned with solvability of the second-order nonlinear neutral delay difference equation $\Delta^{2}\left(x_{n}+a_{n} x_{n-\tau}\right)+$ $\Delta h\left(n, x_{h_{1 n}}, x_{h_{2 n}}, \ldots, x_{h_{k n}}\right)+f\left(n, x_{f_{1 n}}, x_{f_{2 n}}, \ldots, x_{f_{k n}}\right)=b_{n}, \forall n \geq n_{0}$. Utilizing the Banach fixed point theorem and some new techniques, we show the existence of uncountably many unbounded positive solutions for the difference equation, suggest several Mann-type iterative schemes with errors, and discuss the error estimates between the unbounded positive solutions and the sequences generated by the Mann iterative schemes. Four nontrivial examples are given to illustrate the results presented in this paper.

\section{Introduction and Preliminaries}

Recently, the oscillation, nonoscillation, asymptotic behavior, and existence of solutions of different classes of linear and nonlinear second-order difference equations have been studied by many authors; see, for example, [1-26] and the references cited therein. Using the Banach fixed point theorem, Jinfa [5] discussed the existence of a bounded nonoscillatory solution for the second-order neutral delay difference equation with positive and negative coefficients:

$$
\Delta^{2}\left(x_{n}+p x_{n-m}\right)+p_{n} x_{n-k}-q_{n} x_{n-l}=0, \quad \forall n \geq n_{0}
$$

under the condition $p \neq-1$. Luo and Bainov [13] and M. Migda and J. Migda [16] considered the asymptotic behaviors of nonoscillatory solutions for the second-order neutral difference equation with maxima:

$$
\Delta^{2}\left(x_{n}+p_{n} x_{n-k}\right)+q_{n} \max \left\{x_{s}: n-l \leq s \leq n\right\}=0, \quad \forall n \geq 1
$$

and the second-order neutral difference equation:

$$
\Delta^{2}\left(x_{n}+p x_{n-k}\right)+f\left(n, x_{n}\right)=0, \quad \forall n \geq 1,
$$

respectively. Meng and Yan [15] studied the existence of bounded nonoscillatory solutions for the second-order nonlinear nonautonomous neutral delay difference equation:

$$
\Delta^{2}\left(x_{n}-p x_{n-\tau}\right)=\sum_{n=1}^{m} q_{i} x_{n-\sigma_{i}}+f\left(n, x_{n-\eta_{1 n}}, \ldots, x_{n-\eta_{l n}}\right),
$$

$$
\forall n \geq n_{0} .
$$

Applying the cone compression and expansion theorem in Fréchet spaces, Tian and Ge [21] established the existence of multiple positive solutions of the second-order discrete equation on the half-line:

$$
\Delta^{2} x_{n-1}-p \Delta x_{n-1}-q x_{n-1}+f\left(n, x_{n}\right)=0, \quad \forall n \geq 1
$$

with certain boundary value conditions. But to the best of our knowledge, results on multiplicity of unbounded solutions 
for neutral delay difference equations are very scarce in the literature. Nothing has been done with the existence of uncountably many unbounded positive solutions for (1) (5) and any other second-order neutral delay difference equations:

Inspired and motivated by the results in [1-26], in this paper we introduce and study the second-order nonlinear neutral delay difference equation:

$$
\begin{aligned}
& \Delta^{2}\left(x_{n}+a_{n} x_{n-\tau}\right)+\Delta h\left(n, x_{h_{1 n}}, x_{h_{2 n}}, \ldots, x_{h_{k n}}\right) \\
& \quad+f\left(n, x_{f_{1 n}}, x_{f_{2 n}}, \ldots, x_{f_{k n}}\right)=b_{n}, \quad \forall n \geq n_{0},
\end{aligned}
$$

where $\tau, k, n_{0} \in \mathbb{N},\left\{a_{n}\right\}_{n \in \mathbb{N}_{n_{0}}},\left\{b_{n}\right\}_{n \in \mathbb{N}_{n_{0}}} \subset \mathbb{R}, h, f \in C\left(\mathbb{N}_{n_{0}} \times\right.$ $\left.\mathbb{R}^{k}, \mathbb{R}\right),\left\{h_{l n}\right\}_{n \in \mathbb{N}_{n_{0}}},\left\{f_{l n}\right\}_{n \in \mathbb{N}_{n_{0}}} \subseteq \mathbb{N}$, and

$$
\lim _{n \rightarrow \infty} h_{l n}=\lim _{n \rightarrow \infty} f_{l n}=+\infty, \quad l \in\{1,2, \ldots, k\} .
$$

By means of the Banach fixed point theorem and some new techniques, we establish sufficient conditions for the existence of uncountably many unbounded positive solutions of (6), suggest a few Mann iterative schemes with errors for approximating these unbounded positive solutions, and prove their convergence and the error estimates. The results obtained in this paper extend the result in [5]. Four nontrivial examples are interested in the text to illustrate the importance of our results.

Throughout this paper, we assume that $\Delta$ is the forward difference operator defined by $\Delta x_{n}=x_{n+1}-x_{n}, \mathbb{R}=$ $(-\infty,+\infty), \mathbb{R}^{+}=[0,+\infty), \mathbb{Z}, \mathbb{N}_{0}$, and $\mathbb{N}$ denote the sets of all integers, nonnegative integers, and positive integers, respectively,

$$
\begin{gathered}
\mathbb{N}_{t}=\{n: n \in \mathbb{N} \text { with } n \geq t\}, \quad \forall t \in \mathbb{N}, \\
\beta=\min \left\{n_{0}-\tau, \inf \left\{h_{l n}, f_{l n}: 1 \leq l \leq k, n \in \mathbb{N}_{n_{0}}\right\}\right\} \in \mathbb{N}, \\
H_{n}=\max \left\{h_{l n}: l \in\{1,2, \ldots, k\}\right\}, \\
F_{n}=\max \left\{f_{l n}: l \in\{1,2, \ldots, k\}\right\}, \quad \forall n \in \mathbb{N}_{n_{0}},
\end{gathered}
$$

$l_{\beta}^{\infty}$ represents the Banach space of all real sequences on $\mathbb{N}_{\beta}$ with norm

$$
\begin{gathered}
\|x\|=\sup _{n \in \mathbb{N}_{\beta}}\left|\frac{x_{n}}{n}\right|<+\infty \text { for each } x=\left\{x_{n}\right\}_{n \in \mathbb{N}_{\beta}} \in l_{\beta}^{\infty}, \\
A(N, M)=\left\{x=\left\{x_{n}\right\}_{n \in \mathbb{N}_{\beta}} \in l_{\beta}^{\infty}: N \leq \frac{x_{n}}{n} \leq M, n \in \mathbb{N}_{\beta}\right\}
\end{gathered}
$$$$
\text { for any } M>N>0 \text {. }
$$

It is clear that $A(N, M)$ is a closed and convex subset of $l_{\beta}^{\infty}$. By a solution of (6), we mean a sequence $\left\{x_{n}\right\}_{n \in \mathbb{N}_{\beta}}$ with a positive integer $T \geq n_{0}+\tau+\beta$ such that (6) holds for all $n \geq T$.

The following lemmas play important roles in this paper.
Lemma 1 (see [27]). Let $\left\{\alpha_{n}\right\}_{n \in \mathbb{N}_{0}},\left\{\beta_{n}\right\}_{n \in \mathbb{N}_{0}},\left\{\gamma_{n}\right\}_{n \in \mathbb{N}_{0}}$, and $\left\{t_{n}\right\}_{n \in \mathbb{N}_{0}}$ be four nonnegative sequences satisfying the inequality

$$
\alpha_{n+1} \leq\left(1-t_{n}\right) \alpha_{n}+t_{n} \beta_{n}+\gamma_{n}, \quad \forall n \in \mathbb{N}_{0},
$$

where $\left\{t_{n}\right\}_{n \in \mathbb{N}_{0}} \subset[0,1], \sum_{n=0}^{\infty} t_{n}=+\infty, \lim _{n \rightarrow \infty} \beta_{n}=0$, and $\sum_{n=0}^{\infty} \gamma_{n}<+\infty$. Then $\lim _{n \rightarrow \infty} \alpha_{n}=0$.

Lemma 2 (see [11]). Let $\tau, n_{0} \in \mathbb{N}$ and $\left\{b_{n}\right\}_{n \in \mathbb{N}_{n_{0}}}$ be a nonnegative sequence. Then

(a) $\sum_{i=0}^{\infty} \sum_{s=n_{0}+i \tau}^{\infty} b_{s}<+\infty \Leftrightarrow \sum_{s=n_{0}}^{\infty} s b_{s}<+\infty$;

(b) $\sum_{i=0}^{\infty} \sum_{s=n_{0}+i \tau}^{\infty} \sum_{t=s}^{\infty} b_{t}<+\infty \Leftrightarrow \sum_{s=n_{0}}^{\infty} \sum_{t=s}^{\infty} s b_{t}<+\infty$.

\section{Existence of Uncountably Many Unbounded Positive Solutions}

Using the Banach fixed point theorem and the Mann iterative schemes with errors, we next discuss the existence of uncountably many unbounded positive solutions of (6), prove that the Mann iterative schemes with errors converge to these unbounded positive solutions, and compute the error estimates between the Mann iterative schemes with errors and the unbounded positive solutions.

Theorem 3. Assume that there exist two constants $M$ and $N$ with $M>N>0$ and four nonnegative sequences $\left\{P_{n}\right\}_{n \in \mathbb{N}_{n_{0}}},\left\{Q_{n}\right\}_{n \in \mathbb{N}_{n_{0}}},\left\{R_{n}\right\}_{n \in \mathbb{N}_{n_{0}}}$, and $\left\{W_{n}\right\}_{n \in \mathbb{N}_{n_{0}}}$ satisfying

$$
\begin{gathered}
\left|f\left(n, u_{1}, u_{2}, \ldots, u_{k}\right)-f\left(n, \bar{u}_{1}, \bar{u}_{2}, \ldots, \bar{u}_{k}\right)\right| \\
\leq P_{n} \max \left\{\left|u_{l}-\bar{u}_{l}\right|: 1 \leq l \leq k\right\}, \\
\left|h\left(n, u_{1}, u_{2}, \ldots, u_{k}\right)-h\left(n, \bar{u}_{1}, \bar{u}_{2}, \ldots, \bar{u}_{k}\right)\right| \\
\leq R_{n} \max \left\{\left|u_{l}-\bar{u}_{l}\right|: 1 \leq l \leq k\right\}, \\
\forall\left(n, u_{l}, \bar{u}_{l}\right) \in \mathbb{N}_{n_{0}} \times\left(\mathbb{R}^{+} \backslash\{0\}\right)^{2}, \quad 1 \leq l \leq k, \\
\left|f\left(n, u_{1}, u_{2}, \ldots, u_{k}\right)\right| \leq Q_{n}, \quad\left|h\left(n, u_{1}, u_{2}, \ldots, u_{k}\right)\right| \leq W_{n}, \\
\forall\left(n, u_{l}\right) \in \mathbb{N}_{n_{0}} \times\left(\mathbb{R}^{+} \backslash\{0\}\right), \quad 1 \leq l \leq k,
\end{gathered}
$$

$$
\lim _{n \rightarrow \infty} \frac{1}{n} \sum_{i=0}^{\infty} \sum_{s=n+i \tau}^{\infty} \max \left\{R_{s} H_{s}, W_{s}\right\}=0
$$

$$
\begin{gathered}
\lim _{n \rightarrow \infty} \frac{1}{n} \sum_{i=0}^{\infty} \sum_{s=n+i \tau}^{\infty} \sum_{t=s}^{\infty} \max \left\{P_{t} F_{t}, Q_{t},\left|b_{t}\right|\right\}=0, \\
a_{n}=-1 \text { eventually. }
\end{gathered}
$$


Then

(a) for any $L \in(N, M)$, there exist $\theta \in(0,1)$ and $T \geq n_{0}+$ $\tau+\beta$ such that for each $x_{0}=\left\{x_{0 n}\right\}_{n \in \mathbb{N}_{\beta}} \in A(N, M)$, the Mann iterative sequence with errors $\left\{x_{m}\right\}_{m \in \mathbb{N}_{0}}=\left\{x_{m n}\right\}_{(m, n) \in \mathbb{N}_{0} \times \mathbb{N}_{\beta}}$ generated by the scheme

$$
\begin{aligned}
& x_{m+1 n} \\
& \left\{\begin{array}{c}
\left(1-\alpha_{m}-\beta_{m}\right) x_{m n} \\
+\alpha_{m}\{n L
\end{array}\right. \\
& -\sum_{i=1}^{\infty} \sum_{s=n+i \tau}^{\infty}\left[h\left(s, x_{m h_{1 s}}, x_{m h_{2 s}}, \ldots, x_{m h_{k s}}\right)\right. \\
& -\sum_{t=s}^{\infty}\left(f \left(t, x_{m f_{1 t}}, x_{m f_{2 t}}, \ldots,\right.\right. \\
& \left.\left.\left.\left.x_{m f_{k t}}\right)-b_{t}\right)\right]\right\} \\
& =\left\{\begin{array}{c}
+\beta_{m} \gamma_{m n}, \\
\left(1-\alpha_{m}-\beta_{m}\right) x_{m T} \\
+\alpha_{m}\{T L
\end{array}\right. \\
& -\sum_{i=1}^{\infty} \sum_{s=T+i \tau}^{\infty}\left[h\left(s, x_{m h_{1 s}}, x_{m h_{2 s}}, \ldots, x_{m h_{k s}}\right)\right. \\
& -\sum_{t=s}^{\infty}\left(f \left(t, x_{m f_{1 t}}, x_{m f_{2 t}}, \ldots,\right.\right. \\
& \left.\left.\left.\left.x_{m f_{k t}}\right)-b_{t}\right)\right]\right\} \\
& +\beta_{m} \gamma_{m T}, \quad \beta \leq n<T, m \geq 0
\end{aligned}
$$

converges to an unbounded positive solution $x \in A(N, M)$ of (6) and has the following error estimate:

$$
\begin{array}{r}
\left\|x_{m+1}-x\right\| \leq\left[1-(1-\theta) \alpha_{m}\right]\left\|x_{m}-x\right\|+2 M \beta_{m} \\
\forall m \in \mathbb{N}_{0},
\end{array}
$$

where $\left\{\gamma_{m}\right\}_{m \in \mathbb{N}_{0}}=\left\{\gamma_{m n}\right\}_{(m, n) \in \mathbb{N}_{0} \times \mathbb{N}_{\beta}}$ is an arbitrary sequence in $A(N, M)$ and $\left\{\alpha_{m}\right\}_{m \in \mathbb{N}_{0}}$ and $\left\{\beta_{m}\right\}_{m \in \mathbb{N}_{0}}$ are any sequences in $[0,1]$ such that

$$
\sum_{m=0}^{\infty} \alpha_{m}=+\infty
$$

$\sum_{m=0}^{\infty} \beta_{m}<+\infty$ or there exists a sequence

$$
\left\{\xi_{m}\right\}_{m \in \mathbb{N}_{0}} \subset[0,+\infty) \text { satisfying }
$$

$$
\beta_{m}=\xi_{m} \alpha_{m}, \quad m \in \mathbb{N}_{0}, \quad \lim _{m \rightarrow \infty} \xi_{m}=0
$$

(b) equation (6) possesses uncountably many unbounded positive solutions in $A(N, M)$.
Proof. First of all we show that (a) holds. Set $L \in(N, M)$. It follows from (13), (14), and (15) that there exist $\theta \in(0,1)$ and $T \geq n_{0}+\tau+\beta$ satisfying

$$
\begin{gathered}
\theta=\frac{1}{T} \sum_{i=1}^{\infty} \sum_{s=T+i \tau}^{\infty}\left(R_{s} H_{s}+\sum_{t=s}^{\infty} P_{t} F_{t}\right) \\
\frac{1}{T} \sum_{i=1}^{\infty} \sum_{s=T+i \tau}^{\infty}\left(W_{s}+\sum_{t=s}^{\infty} Q_{t}+\sum_{t=s}^{\infty}\left|b_{t}\right|\right)<\min \{M-L, L-N\} \\
a_{n}=-1, \quad \forall n \geq T
\end{gathered}
$$

Define a mapping $S_{L}: A(N, M) \rightarrow l_{\beta}^{\infty}$ by

$$
\begin{aligned}
& S_{L} x_{n} \\
& =\left\{\begin{array}{r}
n L \\
-\sum_{i=1}^{\infty} \sum_{s=n+i \tau}^{\infty}\left\{h\left(s, x_{h_{1 s}}, x_{h_{2 s}}, \ldots, x_{h_{k s}}\right)\right. \\
\left.-\sum_{t=s}^{\infty}\left[f\left(t, x_{f_{1 t}}, x_{f_{2 t}}, \ldots, x_{f_{k t}}\right)-b_{t}\right]\right\} \\
n \geq T, \\
S_{L} x_{T}, \\
\quad \beta \leq n<T
\end{array},\right.
\end{aligned}
$$

for each $x=\left\{x_{n}\right\}_{n \in \mathbb{N}_{\beta}} \in A(N, M)$. In view of (11), (12), (19), (20), and (22), we deduce that for each $x=\left\{x_{n}\right\}_{n \in \mathbb{N}_{\beta}}, y=$ $\left\{y_{n}\right\}_{n \in \mathbb{N}_{\beta}} \in A(N, M)$ and for all $n \geq T$

$$
\begin{aligned}
& \left|\frac{S_{L} x_{n}}{n}-\frac{S_{L} y_{n}}{n}\right| \\
& \leq \frac{1}{n} \sum_{i=1}^{\infty} \sum_{s=n+i \tau}^{\infty}\left[\mid h\left(s, x_{h_{1 s}}, x_{h_{2 s}}, \ldots, x_{h_{k s}}\right)\right. \\
& -h\left(s, y_{h_{1 s}}, y_{h_{2 s}}, \ldots, y_{h_{k s}}\right) \mid \\
& +\sum_{t=s}^{\infty} \mid f\left(t, x_{f_{1 t}}, x_{f_{2 t}}, \ldots, x_{f_{k t}}\right) \\
& \left.-f\left(t, y_{f_{1 t}}, y_{f_{2 t}}, \ldots, y_{f_{k t}}\right) \mid\right] \\
& \leq \frac{1}{n} \sum_{i=1}^{\infty} \sum_{s=n+i \tau}^{\infty}\left[\begin{array}{l}
R_{s} \max \left\{\left|x_{h_{l s}}-y_{h_{l s}}\right|: 1 \leq l \leq k\right\} \\
+\sum_{t=s}^{\infty} P_{t} \max \left\{\left|x_{f_{l t}}-y_{f_{l t}}\right|: 1 \leq l \leq k\right\}
\end{array}\right]
\end{aligned}
$$




$$
\begin{aligned}
& \leq \frac{\|x-y\|}{n} \sum_{i=1}^{\infty} \sum_{s=n+i \tau}^{\infty}\left[R_{s} \max \left\{h_{l s}: 1 \leq l \leq k\right\}\right. \\
& \left.+\sum_{t=s}^{\infty} P_{t} \max \left\{f_{l t}: 1 \leq l \leq k\right\}\right] \\
& \leq \frac{\|x-y\|}{T} \sum_{i=1}^{\infty} \sum_{s=T+i \tau}^{\infty}\left(R_{s} H_{s}+\sum_{t=s}^{\infty} P_{t} F_{t}\right) \\
& =\theta\|x-y\|, \\
& \left|\frac{S_{L} x_{n}}{n}-L\right| \\
& =\mid \frac{1}{n} \sum_{i=1}^{\infty} \sum_{s=n+i \tau}^{\infty}\left\{h\left(s, x_{h_{1 s}}, x_{h_{2 s}}, \ldots, x_{h_{k s}}\right)\right. \\
& \left.-\sum_{t=s}^{\infty}\left[f\left(t, x_{f_{1 t}}, x_{f_{2 t}}, \ldots, x_{f_{k t}}\right)-b_{t}\right]\right\} \mid \\
& \leq \frac{1}{n} \sum_{i=1}^{\infty} \sum_{s=n+i \tau}^{\infty}\left\{\left|h\left(s, x_{h_{1 s}}, x_{h_{2 s}}, \ldots, x_{h_{k s}}\right)\right|\right. \\
& \left.+\sum_{t=s}^{\infty}\left[\left|f\left(t, x_{f_{1 t}}, x_{f_{2 t}}, \ldots, x_{f_{k t}}\right)\right|+\left|b_{t}\right|\right]\right\} \\
& \leq \frac{1}{T} \sum_{i=1}^{\infty} \sum_{s=T+i \tau}^{\infty}\left[W_{s}+\sum_{t=s}^{\infty}\left(Q_{t}+\left|b_{t}\right|\right)\right] \\
& <\min \{M-L, L-N\} \text {, }
\end{aligned}
$$

which yield that

$S_{L}(A(N, M)) \subseteq A(N, M), \quad\left\|S_{L} x-S_{L} y\right\| \leq \theta\|x-y\|$, $\forall x, y \in A(N, M)$,

which means that $S_{L}$ is a contraction in $A(N, M)$. It follows from the Banach fixed point theorem that $S_{L}$ has a unique fixed point $x=\left\{x_{n}\right\}_{n \in \mathbb{N}_{\beta}} \in A(N, M)$, that is,

$x_{n}=n L$

$$
\begin{aligned}
&-\sum_{i=1}^{\infty} \sum_{s=n+i \tau}^{\infty}\left\{h\left(s, x_{h_{1 s}}, x_{h_{2 s}}, \ldots, x_{h_{k s}}\right)\right. \\
&\left.-\sum_{t=s}^{\infty}\left[f\left(t, x_{f_{1 t}}, x_{f_{2 t}}, \ldots, x_{f_{k t}}\right)-b_{t}\right]\right\}, \\
& \forall n \geq T,
\end{aligned}
$$

$$
\begin{aligned}
& x_{n-\tau} \\
& =(n-\tau) L \\
& \quad-\sum_{i=1}^{\infty} \sum_{s=n+(i-1) \tau}^{\infty}\left\{h\left(s, x_{h_{1 s}}, x_{h_{2 s}}, \ldots, x_{h_{k s}}\right)\right. \\
& \left.\quad-\sum_{t=s}^{\infty}\left[f\left(t, x_{f_{1 t}}, x_{f_{2 t}}, \ldots, x_{f_{k t}}\right)-b_{t}\right]\right\}, \\
& \forall n \geq T+\tau,
\end{aligned}
$$

which imply that

$$
\begin{array}{r}
x_{n}-x_{n-\tau} \\
=\tau L+\sum_{s=n}^{\infty}\left\{h\left(s, x_{h_{1 s}}, x_{h_{2 s}}, \ldots, x_{h_{k s}}\right)\right. \\
\left.-\sum_{t=s}^{\infty}\left[f\left(t, x_{f_{1 t}}, x_{f_{2 t}}, \ldots, x_{f_{k t}}\right)-b_{t}\right]\right\}, \\
\forall n \geq T+\tau,
\end{array}
$$

which yields that

$$
\begin{aligned}
& \Delta\left(x_{n}-x_{n-\tau}\right)=-h\left(n, x_{h_{1 n}}, x_{h_{2 n}}, \ldots, x_{h_{k n}}\right) \\
&+\sum_{t=n}^{\infty}\left[f\left(t, x_{f_{1 t}}, x_{f_{2 t}}, \ldots, x_{f_{k t}}\right)-b_{t}\right], \\
& \forall n \geq T+\tau, \\
& \Delta^{2}\left(x_{n}-x_{n-\tau}\right)=-\Delta h\left(n, x_{h_{1 n}}, x_{h_{2 n}}, \ldots, x_{h_{k n}}\right) \\
&-f\left(n, x_{f_{1 n}}, x_{f_{2 n}}, \ldots, x_{f_{k n}}\right)+b_{n}, \\
& \forall n \geq T+\tau,
\end{aligned}
$$

which together with (21) gives that $x=\left\{x_{n}\right\}_{n \in \mathbb{N}_{\beta}}$ is an unbounded positive solution of (6) in $A(N, M)$. It follows from (16), (19), (21), (22), and (24) that for any $m \in \mathbb{N}_{0}$ and $n \geq T$

$$
\begin{aligned}
& \left|\frac{x_{m+1 n}}{n}-\frac{x_{n}}{n}\right| \\
& =\frac{1}{n} \mid\left(1-\alpha_{m}-\beta_{m}\right) x_{m n}
\end{aligned}
$$




$$
\begin{aligned}
& +\alpha_{m}\{n L \\
& -\sum_{i=1}^{\infty} \sum_{s=n+i \tau}^{\infty}\left[h\left(s, x_{m h_{1 s}}, x_{m h_{2 s}}, \ldots, x_{m h_{k s}}\right)\right. \\
& \quad-\sum_{t=s}^{\infty}\left(f \left(t, x_{m f_{1 t}}, x_{m f_{2 t}}, \ldots,\right.\right. \\
& +\beta_{m} \gamma_{m n}-x_{n} \mid \\
& \left.\left.\left.\left.\leq\left(1-\alpha_{m}-\beta_{m}\right) \frac{\left|x_{m n}-x_{n}\right|}{n}\right)-b_{t}\right)\right]\right\} \\
& +\alpha_{m} \frac{\left|S_{L} x_{m n}-S_{L} x_{n}\right|}{n}+\beta_{m} \frac{\left|\gamma_{m n}-x_{n}\right|}{n} \\
& \leq\left(1-\alpha_{m}-\beta_{m}\right)\left\|x_{m}-x\right\|+\theta \alpha_{m}\left\|x_{m}-x\right\|+2 M \beta_{m} \\
& \leq\left[1-(1-\theta) \alpha_{m}\right]\left\|x_{m}-x\right\|+2 M \beta_{m},
\end{aligned}
$$

which implies that

$$
\begin{array}{r}
\left\|x_{m+1}-x\right\| \leq\left[1-(1-\theta) \alpha_{m}\right]\left\|x_{m}-x\right\| \\
\quad 2 M \beta_{m} \\
\forall m \in \mathbb{N}_{0} .
\end{array}
$$

That is, (17) holds. Thus, Lemma 1 and (17) and (18) guarantee that $\lim _{m \rightarrow \infty} x_{m}=x$.

Next we show that (b) holds. Let $L_{1}, L_{2} \in(N, M)$ and $L_{1} \neq L_{2}$. As in the proof of (a), we deduce similarly that for each $c \in\{1,2\}$ there exist constants $\theta_{c} \in(0,1), T_{c} \geq n_{0}+\tau+$ $\beta$, and a mapping $S_{L_{c}}$ satisfying (19) $\sim(24)$, where $\theta, L$, and $T$ are replaced by $\theta_{c}, L_{c}$ and $T_{c}$, respectively, and the mapping $S_{L_{c}}$ has a fixed point $z^{c}=\left\{z_{n}^{c}\right\}_{n \in \mathbb{N}_{\beta}} \in A(N, M)$, which is an unbounded positive solution of $(6)$ in $A(N, M)$, that is,

$$
\begin{array}{r}
z_{n}^{c}=n L_{c} \\
-\sum_{i=1}^{\infty} \sum_{s=n+i \tau}^{\infty}\left\{h\left(s, z_{h_{1 s}}^{c}, z_{h_{2 s}}^{c}, \ldots, z_{h_{k s}}^{c}\right)\right. \\
\left.\quad-\sum_{t=s}^{\infty}\left[f\left(t, z_{f_{1 t}}^{c}, z_{f_{2 t}}^{c}, \ldots, z_{f_{k t}}^{c}\right)-b_{t}\right]\right\}, \\
\forall n \geq T_{c},
\end{array}
$$

which together with (11) and (17) implies that for $n \geq$ $\max \left\{T_{1}, T_{2}\right\}$

$$
\begin{aligned}
& \left|\frac{z_{n}^{1}}{n}-\frac{z_{n}^{2}}{n}\right| \\
& \geq\left|L_{1}-L_{2}\right| \\
& -\frac{1}{n} \sum_{i=1}^{\infty} \sum_{s=n+i \tau}^{\infty}\left\{\mid h\left(s, z_{h_{1 s}}^{1}, z_{h_{2 s}}^{1}, \ldots, z_{h_{k s}}^{1}\right)\right. \\
& -h\left(s, z_{h_{1 s}}^{2}, z_{h_{2 s}}^{2}, \ldots, z_{h_{k s}}^{2}\right) \mid \\
& +\sum_{t=s}^{\infty} \mid f\left(t, z_{f_{1 t}}^{1}, z_{f_{2 t}}^{1}, \ldots, z_{f_{k t}}^{1}\right) \\
& \geq\left|L_{1}-L_{2}\right| \\
& \left.-f\left(t, z_{f_{1 t}}^{2}, z_{f_{2 t}}^{2}, \ldots, z_{f_{k t}}^{2}\right) \mid\right\} \\
& -\frac{1}{n} \sum_{i=1}^{\infty} \sum_{s=n+i \tau}^{\infty}\left[R_{s} \max \left\{\left|z_{h_{l s}}^{1}-z_{h_{l s}}^{2}\right|: 1 \leq l \leq k\right\}\right. \\
& \left.+\sum_{t=s}^{\infty} P_{t} \max \left\{\left|z_{f_{l t}}^{1}-z_{f_{l t}}^{2}\right|: 1 \leq l \leq k\right\}\right] \\
& \geq\left|L_{1}-L_{2}\right|-\frac{\left\|z^{1}-z^{2}\right\|}{n} \sum_{i=1}^{\infty} \sum_{s=n+i \tau}^{\infty}\left(R_{s} H_{s}+\sum_{t=s}^{\infty} P_{t} F_{t}\right) \\
& \geq\left|L_{1}-L_{2}\right|-\frac{\left\|z^{1}-z^{2}\right\|}{\max \left\{T_{1}, T_{2}\right\}} \\
& \times \sum_{i=1}^{\infty} \sum_{s=\max \left\{T_{1}, T_{2}\right\}+i \tau}^{\infty}\left(R_{s} H_{s}+\sum_{t=s}^{\infty} P_{t} F_{t}\right) \\
& \geq\left|L_{1}-L_{2}\right|-\max \left\{\theta_{1}, \theta_{2}\right\}\left\|z^{1}-z^{2}\right\| \text {, }
\end{aligned}
$$

which yields that

$$
\left\|z^{1}-z^{2}\right\| \geq \frac{\left|L_{1}-L_{2}\right|}{1+\max \left\{\theta_{1}, \theta_{2}\right\}}>0,
$$

that is, $z^{1} \neq z^{2}$. This completes the proof.

Theorem 4. Assume that there exist two constants $M$ and $N$ with $M>N>0$ and four nonnegative sequences $\left\{P_{n}\right\}_{n \in \mathbb{N}_{n_{0}}}$, $\left\{Q_{n}\right\}_{n \in \mathbb{N}_{n_{0}}},\left\{R_{n}\right\}_{n \in \mathbb{N}_{n_{0}}}$, and $\left\{W_{n}\right\}_{n \in \mathbb{N}_{n_{0}}}$ satisfying (11), (12),

$$
\begin{gathered}
\lim _{n \rightarrow \infty} \frac{1}{n} \sum_{s=n}^{\infty} \max \left\{R_{s} H_{s}, W_{s}\right\}=0, \\
\lim _{n \rightarrow \infty} \frac{1}{n} \sum_{s=n}^{\infty} \sum_{t=s}^{\infty} \max \left\{P_{t} F_{t}, Q_{t},\left|b_{t}\right|\right\}=0,
\end{gathered}
$$

$a_{n}=1 \quad$ eventually 
Then

(a) for any $L \in(N, M)$, there exist $\theta \in(0,1)$ and $T \geq n_{0}+$ $\tau+\beta$ such that for each $x_{0}=\left\{x_{0 n}\right\}_{n \in \mathbb{N}_{\beta}} \in A(N, M)$, the Mann iterative sequence with errors $\left\{x_{m}\right\}_{m \in \mathbb{N}_{0}}=\left\{x_{m n}\right\}_{(m, n) \in \mathbb{N}_{0} \times \mathbb{N}_{\beta}}$ generated by the scheme

$$
\begin{aligned}
& x_{m+1 n} \\
& \left\{\begin{array}{c}
\left(1-\alpha_{m}-\beta_{m}\right) x_{m n} \\
+\alpha_{m}\{n L
\end{array}\right. \\
& +\sum_{i=1}^{\infty} \sum_{s=n+(2 i-1) \tau}^{n+2 i \tau-1}\left[h\left(s, x_{m h_{1 s}}, x_{m h_{2 s}}, \ldots, x_{m h_{k s}}\right)\right. \\
& -\sum_{t=s}^{\infty}\left(f \left(t, x_{m f_{1 t}}, x_{m f_{2 t}}, \ldots,\right.\right. \\
& =\left\{\begin{array}{cr} 
& \left.\left.\left.\left.x_{m f_{k t}}\right)-b_{t}\right)\right]\right\} \\
+\beta_{m} \gamma_{m n}, & n \geq T, m \geq 0, \\
\left(1-\alpha_{m}-\beta_{m}\right) x_{m T} & \\
+\alpha_{m}\{T L & \\
& +\sum_{i=1}^{\infty} \sum_{s=T+(2 i-1) \tau}^{T+2 i \tau-1}\left[h\left(s, x_{m h_{1 s}}, x_{m h_{2 s}}, \ldots, x_{m h_{k s}}\right)\right.
\end{array}\right. \\
& =\left\{\begin{array}{cc} 
& \\
+\beta_{m} \gamma_{m n}, & n \geq T, m \geq 0, \\
\left.\left.\left.\left.\left(1-\alpha_{m}-\beta_{m}\right) x_{m T}\right)-b_{t}\right)\right]\right\} & \\
+\alpha_{m}\{T L & \\
+\sum_{i=1}^{\infty} \sum_{s=T+(2 i-1) \tau}^{T+2 i \tau-1}\left[h\left(s, x_{m h_{1 s}}, x_{m h_{2 s}}, \ldots, x_{m h_{k s}}\right)\right.
\end{array}\right. \\
& -\sum_{t=s}^{\infty}\left(f \left(t, x_{m f_{1 t}}, x_{m f_{2 t}}, \ldots,\right.\right. \\
& \left.\left.\left.\left.x_{m f_{k t}}\right)-b_{t}\right)\right]\right\} \\
& +\beta_{m} \gamma_{m T}, \quad \beta \leq n<T, m \geq 0
\end{aligned}
$$

converges to an unbounded positive solution $x \in A(N, M)$ of (6) and has the error estimate (17), where $\left\{\gamma_{m}\right\}_{m \in \mathbb{N}_{0}}=$ $\left\{\gamma_{m n}\right\}_{(m, n) \in \mathbb{N}_{0} \times \mathbb{N}_{\beta}}$ is an arbitrary sequence in $A(N, M)$, and $\left\{\alpha_{m}\right\}_{m \in \mathbb{N}_{0}}$ and $\left\{\beta_{m}\right\}_{m \in \mathbb{N}_{0}}$ are any sequences in $[0,1]$ satisfying (18);

(b) equation (6) possesses uncountably many unbounded positive solutions in $A(N, M)$.

Proof. Let $L \in(N, M)$. It follows from (33) (35) that there exist $\theta \in(0,1)$ and $T \geq n_{0}+\tau+\beta$ satisfying

$$
\begin{gathered}
\theta=\frac{1}{T} \sum_{s=T}^{\infty}\left(R_{s} H_{s}+\sum_{t=s}^{\infty} P_{t} F_{t}\right), \\
\frac{1}{T} \sum_{s=T}^{\infty}\left(W_{s}+\sum_{t=s}^{\infty}\left(Q_{t}+\left|b_{t}\right|\right)\right)<\min \{M-L, L-N\}, \\
a_{n}=1, \quad \forall n \geq T .
\end{gathered}
$$

Define a mapping $S_{L}: A(N, M) \rightarrow l_{\beta}^{\infty}$ by

$$
\begin{aligned}
& S_{L} x_{n} \\
& =\left\{\begin{array}{r}
n L \\
+\sum_{i=1}^{\infty} \sum_{s=n+(2 i-1) \tau}^{n+2 i \tau-1}\left\{h\left(s, x_{h_{1 s}}, x_{h_{2 s}}, \ldots, x_{h_{k s}}\right)\right. \\
\left.-\sum_{t=s}^{\infty}\left[f\left(t, x_{f_{1 t}}, x_{f_{2 t}}, \ldots, x_{f_{k t}}\right)-b_{t}\right]\right\}, \\
n \geq T, \\
S_{L} x_{T}, \\
\beta \leq n<T
\end{array}\right.
\end{aligned}
$$

for each $x=\left\{x_{n}\right\}_{n \in \mathbb{N}_{\beta}} \in A(N, M)$. Using (11), (12), (37), (38), and (40), we get that for each $x=\left\{x_{n}\right\}_{n \in \mathbb{N}_{\beta}}, y=\left\{y_{n}\right\}_{n \in \mathbb{N}_{\beta}} \in$ $A(N, M)$ and $n \geq T$

$$
\begin{aligned}
& \left|\frac{S_{L} x_{n}}{n}-\frac{S_{L} y_{n}}{n}\right| \\
& \leq \frac{1}{n} \sum_{i=1}^{\infty} \sum_{s=n+(2 i-1) \tau}^{n+2 i \tau-1}\left[\mid h\left(s, x_{h_{1 s}}, x_{h_{2 s}}, \ldots, x_{h_{k s}}\right)\right. \\
& -h\left(s, y_{h_{1 s}}, y_{h_{2 s}}, \ldots, y_{h_{k s}}\right) \mid \\
& +\sum_{t=s}^{\infty} \mid f\left(t, x_{f_{1 t}}, x_{f_{2 t}}, \ldots, x_{f_{k t}}\right) \\
& \left.-f\left(t, y_{f_{1 t}}, y_{f_{2 t}}, \ldots, y_{f_{k t}}\right) \mid\right] \\
& \leq \frac{\|x-y\|}{n} \sum_{i=1}^{\infty} \sum_{s=n+(2 i-1) \tau}^{n+2 i \tau-1}\left(R_{s} H_{s}+\sum_{t=s}^{\infty} P_{t} F_{t}\right) \\
& \leq \frac{\|x-y\|}{T} \sum_{s=T}^{\infty}\left(R_{s} H_{s}+\sum_{t=s}^{\infty} P_{t} F_{t}\right) \\
& =\theta\|x-y\|, \\
& \left|\frac{S_{L} x_{n}}{n}-L\right| \\
& \leq \frac{1}{n} \\
& \times \sum_{i=1}^{\infty} \sum_{s=n+(2 i-1) \tau}^{n+2 i \tau-1}\left\{\left|h\left(s, x_{h_{1 s}}, x_{h_{2 s}}, \ldots, x_{h_{k s}}\right)\right|\right. \\
& \left.+\sum_{t=s}^{\infty}\left[\left|f\left(t, x_{f_{1 t}}, x_{f_{2 t}}, \ldots, x_{f_{k t}}\right)\right|+\left|b_{t}\right|\right]\right\} \\
& \leq \frac{1}{T} \sum_{i=T}^{\infty}\left[W_{s}+\sum_{t=s}^{\infty}\left(Q_{t}+\left|b_{t}\right|\right)\right] \\
& <\min \{M-L, L-N\} \text {, }
\end{aligned}
$$


which imply (24). Consequently, (24) means that $S_{L}$ is a contraction in $A(N, M)$ and has a unique fixed point $x=$ $\left\{x_{n}\right\}_{n \in \mathbb{N}_{\beta}} \in A(N, M)$, which is also an unbounded positive solution of (6) in $A(N, M)$. The rest of the proof is similar to the proof of Theorem 3 and is omitted. This completes the proof.

Theorem 5. Assume that there exist three constants $a, M$, and $N$ with $(1-a) M>N>0$ and four nonnegative sequences $\left\{P_{n}\right\}_{n \in \mathbb{N}_{n_{0}}},\left\{Q_{n}\right\}_{n \in \mathbb{N}_{n_{0}}},\left\{R_{n}\right\}_{n \in \mathbb{N}_{n_{0}}}$, and $\left\{W_{n}\right\}_{n \in \mathbb{N}_{n_{0}}}$ satisfying (11), (12), (33), (34) and

$$
0 \leq a_{n} \leq a<1 \quad \text { eventually. }
$$

Then

(a) for any $L \in(a M+N, M)$, there exist $\theta \in(0,1)$ and $T \geq n_{0}+\tau+\beta$ such that for any $x_{0}=\left\{x_{0 n}\right\}_{n \in \mathbb{N}_{\beta}} \in$ $A(N, M)$, the Mann iterative sequence with errors $\left\{x_{m}\right\}_{m \in \mathbb{N}_{0}}=$ $\left\{x_{m n}\right\}_{(m, n) \in \mathbb{N}_{0} \times \mathbb{N}_{\beta}}$ generated by the scheme

$$
\begin{aligned}
& x_{m+1 n}
\end{aligned}
$$

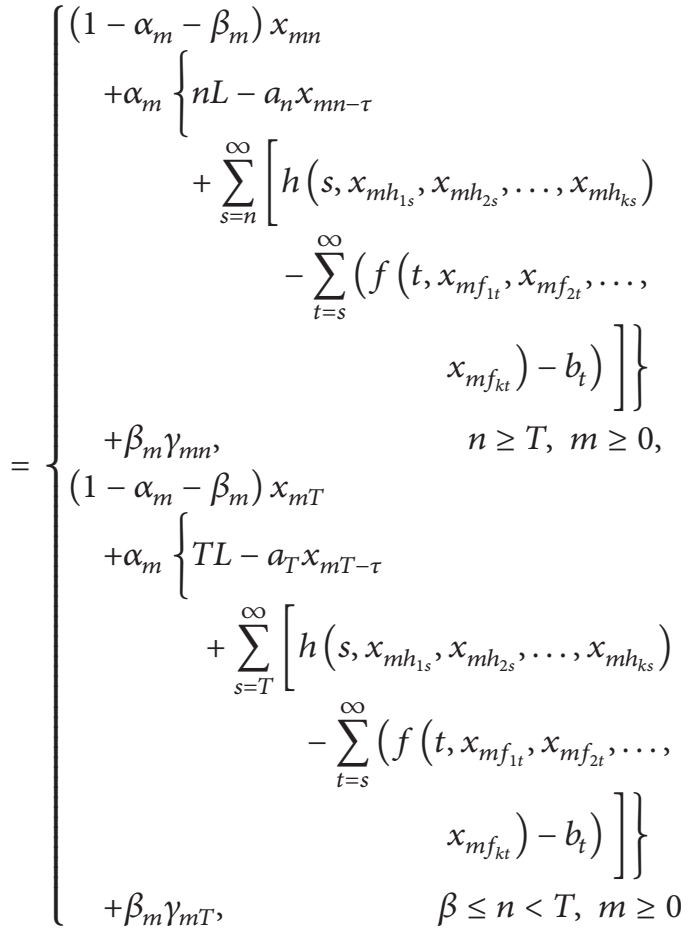

converges to an unbounded positive solution $x \in A(N, M)$ of (6) and has the error estimate (17), where $\left\{\gamma_{m}\right\}_{m \in \mathbb{N}_{0}}=$ $\left\{\gamma_{m n}\right\}_{(m, n) \in \mathbb{N}_{0} \times \mathbb{N}_{\beta}}$ is an arbitrary sequence in $A(N, M)$ and $\left\{\alpha_{m}\right\}_{m \in \mathbb{N}_{0}}$ and $\left\{\beta_{m}\right\}_{m \in \mathbb{N}_{0}}$ are any sequences in $[0,1]$ satisfying (18);

(b) equation (6) possesses uncountably many unbounded positive solutions in $A(N, M)$.
Proof. Put $L \in(a M+N, M)$. It follows from (33), (34), and (42) that there exist $\theta \in(0,1)$ and $T \geq n_{0}+\tau+\beta$ satisfying

$$
\begin{gathered}
\theta=a+\frac{1}{T} \sum_{s=T}^{\infty}\left(R_{s} H_{s}+\sum_{t=s}^{\infty} P_{t} F_{t}\right), \\
\frac{1}{T} \sum_{s=T}^{\infty}\left[W_{s}+\sum_{t=s}^{\infty}\left(Q_{t}+\left|b_{t}\right|\right)\right]<\min \{M-L, L-a M-N\}, \\
0 \leq a_{n} \leq a<1, \quad \forall n \geq T .
\end{gathered}
$$

Define a mapping $S_{L}: A(N, M) \rightarrow l_{\beta}^{\infty}$ by

$S_{L} x_{n}$

$$
= \begin{cases}n L-a_{n} x_{n-\tau} & \\ +\sum_{s=n}^{\infty}\left\{h\left(s, x_{h_{1 s}}, x_{h_{2 s}}, \ldots, x_{h_{k s}}\right)\right. & \\ \left.-\sum_{t=s}^{\infty}\left[f\left(t, x_{f_{1 t}}, x_{f_{2 t}}, \ldots, x_{f_{k t}}\right)-b_{t}\right]\right\}, & n \geq T, \\ S_{L} x_{T}, & \beta \leq n<T,\end{cases}
$$

for each $x=\left\{x_{n}\right\}_{n \in \mathbb{N}_{\beta}} \in A(N, M)$. In view of (11), (12), and (44) and (45), we obtain that for each $x=\left\{x_{n}\right\}_{n \in \mathbb{N}_{\beta}}, y=$ $\left\{y_{n}\right\}_{n \in \mathbb{N}_{\beta}} \in A(N, M)$ and $n \geq T$,

$$
\begin{aligned}
& \left|\frac{S_{L} x_{n}}{n}-\frac{S_{L} y_{n}}{n}\right| \\
& \leq a_{n}\left|\frac{x_{n-\tau}-y_{n-\tau}}{n}\right| \\
& +\frac{1}{n} \sum_{s=n}^{\infty}\left[\mid h\left(s, x_{h_{1 s}}, x_{h_{2 s}}, \ldots, x_{h_{k s}}\right)\right. \\
& \quad-h\left(s, y_{h_{1 s}}, y_{h_{2 s}}, \ldots, y_{h_{k s}}\right) \mid \\
& \quad+\sum_{t=s}^{\infty} \mid f\left(t, x_{f_{1 t}}, x_{f_{2 t}}, \ldots, x_{f_{k t}}\right) \\
& \leq\left[\begin{array}{c}
\left.a+\frac{1}{T} \sum_{s=T}^{\infty}\left(R_{s} H_{s}+\sum_{t=s}^{\infty} P_{t} F_{t}\right)\right]\|x-y\| \\
\left.=\theta\|x-y\|, \quad f\left(t, y_{f_{1 t}}, y_{f_{2 t}}, \ldots, y_{f_{k t}}\right) \mid\right]
\end{array}\right.
\end{aligned}
$$




$$
\begin{aligned}
& \frac{S_{L} x_{n}}{n} \leq L \\
& +\frac{1}{n} \sum_{s=n}^{\infty}\left\{\left|h\left(s, x_{h_{1 s}}, x_{h_{2 s}}, \ldots, x_{h_{k s}}\right)\right|\right. \\
& \left.+\sum_{t=s}^{\infty}\left[\left|f\left(t, x_{f_{1 t}}, x_{f_{2 t}}, \ldots, x_{f_{k t}}\right)\right|+\left|b_{t}\right|\right]\right\} \\
& \leq L+\frac{1}{T} \sum_{s=T}^{\infty}\left[W_{s}+\sum_{t=s}^{\infty}\left(Q_{t}+\left|b_{t}\right|\right)\right] \\
& <L+\min \{M-L, L-a M-N\} \\
& \leq M \text {, } \\
& \frac{S_{L} x_{n}}{n} \geq L-a M \\
& -\frac{1}{n} \sum_{s=n}^{\infty}\left\{\left|h\left(s, x_{h_{1 s}}, x_{h_{2 s}}, \ldots, x_{h_{k s}}\right)\right|\right. \\
& \left.+\sum_{t=s}^{\infty}\left[\left|f\left(t, x_{f_{1 t}}, x_{f_{2 t}}, \ldots, x_{f_{k t}}\right)\right|+\left|b_{t}\right|\right]\right\} \\
& \geq L-a M-\frac{1}{T} \sum_{s=T}^{\infty}\left[W_{s}+\sum_{t=s}^{\infty}\left(Q_{t}+\left|b_{t}\right|\right)\right] \\
& >L-a M-\min \{M-L, L-a M-N\} \\
& \geq N \text {, }
\end{aligned}
$$

which give (24), in turn, which implies that $S_{L}$ is a contraction in $A(N, M)$ and possesses a unique fixed point $x=\left\{x_{n}\right\}_{n \in \mathbb{N}_{\beta}} \in$ $A(N, M)$, which is an unbounded positive solution of (6) in $A(N, M)$. The rest of the proof is similar to that of Theorem 3 and is omitted. This completes the proof.

Theorem 6. Assume that there exist constants a, $M$, and $N$ with $(1+a) M>N>0$ and four nonnegative sequences $\left\{P_{n}\right\}_{n \in \mathbb{N}_{n_{0}}},\left\{Q_{n}\right\}_{n \in \mathbb{N}_{n_{0}}},\left\{R_{n}\right\}_{n \in \mathbb{N}_{n_{0}}}$, and $\left\{W_{n}\right\}_{n \in \mathbb{N}_{n_{0}}}$ satisfying (11), (12), (33), (34), and

$$
-1<a \leq a_{n} \leq 0 \quad \text { eventually. }
$$

Then

(a) for any $L \in(N,(1+a) M)$, there exist $\theta \in(0,1)$ and $T \geq n_{0}+\tau+\beta$ such that for any $x_{0}=\left\{x_{0 n}\right\}_{n \in \mathbb{N}_{\beta}} \in A(N, M)$ and the Mann iterative sequence with errors $\left\{x_{m}\right\}_{m \in \mathbb{N}_{0}}=$ $\left\{x_{m n}\right\}_{(m, n) \in \mathbb{N}_{0} \times \mathbb{N}_{\beta}}$ generated by (43) converges to an unbounded positive solution $x \in A(N, M)$ of (6) and has the error estimate (17), where $\left\{\gamma_{m}\right\}_{m \in \mathbb{N}_{0}}=\left\{\gamma_{m n}\right\}_{(m, n) \in \mathbb{N}_{0} \times \mathbb{N}_{\beta}}$ is an arbitrary sequence in $A(N, M),\left\{\alpha_{m}\right\}_{m \in \mathbb{N}_{0}}$ and $\left\{\beta_{m}\right\}_{m \in \mathbb{N}_{0}}$ are any sequences in $[0,1]$ satisfying $(18)$;

(b) equation (6) possesses uncountably many unbounded positive solutions in $A(N, M)$.
Proof. Put $L \in(N,(1+a) M)$. It follows from (33), (34), and (47) that there exist $\theta \in(0,1)$ and $T \geq n_{0}+\tau+\beta$ satisfying

$$
\begin{gathered}
\theta=-a+\frac{1}{T} \sum_{s=T}^{\infty}\left(R_{s} H_{s}+\sum_{t=s}^{\infty} P_{t} F_{t}\right), \\
\frac{1}{T} \sum_{s=T}^{\infty}\left[W_{s}+\sum_{t=s}^{\infty}\left(Q_{t}+\left|b_{t}\right|\right)\right]<\min \{(1+a) M-L, L-N\},
\end{gathered}
$$

$$
-1<a \leq a_{n} \leq 0, \quad \forall n \geq T .
$$

Define a mapping $S_{L}: A(N, M) \rightarrow l_{\beta}^{\infty}$ by (45). By virtue of (12), (45), (48), and (50), we easily verify that

$$
\begin{aligned}
& \frac{S_{L} x_{n}}{n} \leq L-a M \\
& +\frac{1}{n} \sum_{s=n}^{\infty}\left\{\left|h\left(s, x_{h_{1 s}}, x_{h_{2 s}}, \ldots, x_{h_{k s}}\right)\right|\right. \\
& \left.+\sum_{t=s}^{\infty}\left[\left|f\left(t, x_{f_{1 t}}, x_{f_{2 t}}, \ldots, x_{f_{k t}}\right)\right|+\left|b_{t}\right|\right]\right\} \\
& \leq L-a M+\frac{1}{T} \sum_{s=T}^{\infty}\left[W_{s}+\sum_{t=s}^{\infty}\left(Q_{t}+\left|b_{t}\right|\right)\right] \\
& <L+\min \{(1+a) M-L, L-N\} \\
& \leq M \text {, } \\
& \frac{S_{L} x_{n}}{n} \geq L \\
& -\frac{1}{n} \sum_{s=T}^{n-1}\left\{\left|h\left(s, x_{h_{1 s}}, x_{h_{2 s}}, \ldots, x_{h_{k s}}\right)\right|\right. \\
& \left.+\sum_{t=s}^{\infty}\left[\left|f\left(t, x_{f_{1 t}}, x_{f_{2 t}}, \ldots, x_{f_{k t}}\right)\right|+\left|b_{t}\right|\right]\right\} \\
& \geq L-\frac{1}{T} \sum_{s=T}^{\infty}\left[W_{s}+\sum_{t=s}^{\infty}\left(Q_{t}+\left|b_{t}\right|\right)\right] \\
& >L-\min \{(1+a) M-L, L-N\} \\
& \geq N \text {, }
\end{aligned}
$$

which yield that $S_{L}(A(N, M)) \subseteq A(N, M)$. The rest of the proof is similar to that of Theorem 5 and is omitted. This completes the proof.

Remark 7. Theorems 3 6 extend and improve Theorem 1 in [5].

\section{Examples}

In this section we suggest four examples to explain the results presented in Section 2. Note that Theorem 1 in [5] is useless for all these examples. 
Example 8. Consider the second-order nonlinear neutral delay difference equation:

$$
\begin{gathered}
\Delta^{2}\left(x_{n}-x_{n-\tau}\right)+\Delta\left(\frac{\sin ^{2} x_{n-2}}{n^{4}}\right)+\frac{1}{\left(n^{6}+n^{3}+2\right)\left(1+\left|x_{n^{2}}\right|^{3}\right)} \\
=\frac{n^{2}-3 n+1}{n^{7}+n^{4}+1}, \quad n \geq 3,
\end{gathered}
$$

where $\tau \in \mathbb{N}$ is fixed. Let $n_{0}=3, k=1, \beta=\min \{3-\tau, 1\}, M$ and $N$ two positive constants with $M>N$ and

$$
\begin{gathered}
a_{n}=-1, \quad b_{n}=\frac{n^{2}-3 n+1}{n^{7}+n^{4}+1}, \\
f(n, u)=\frac{1}{\left(n^{6}+n^{3}+2\right)\left(1+|u|^{3}\right)}, \\
h(n, u)=\frac{\sin ^{2} u}{n^{4}}, \quad F_{n}=f_{1 n}=n^{2}, \quad H_{n}=h_{1 n}=n-2, \\
P_{n}=\frac{3 M^{2}}{\left(1+N^{3}\right)^{2} n^{6}}, \quad Q_{n}=\frac{1}{n^{6}}, \quad R_{n}=\frac{2}{n^{4}}, \\
W_{n}=\frac{1}{n^{4}}, \quad \forall(n, u) \in \mathbb{N}_{n_{0}} \times \mathbb{R} .
\end{gathered}
$$

It is easy to see that (11), (12), and (15) are satisfied. Note that

$$
\begin{aligned}
\sum_{s=n}^{\infty} s & \max \left\{R_{s} H_{s}, W_{s}\right\} \\
& =\sum_{s=n}^{\infty} s \max \left\{\frac{2(s-2)}{s^{4}}, \frac{1}{s^{4}}\right\} \\
& =\sum_{s=n}^{\infty} \frac{2(s-2)}{s^{3}}<+\infty, \quad \forall n \in \mathbb{N}_{n_{0}},
\end{aligned}
$$

$$
\begin{aligned}
& \sum_{s=n}^{\infty} \sum_{t=s}^{\infty} s \max \left\{P_{t} F_{t}, Q_{t},\left|b_{t}\right|\right\} \\
& \quad=\sum_{s=n}^{\infty} \sum_{t=s}^{\infty} s \max \left\{\frac{3 M^{2}}{\left(1+N^{3}\right)^{2} t^{4}}, \frac{1}{t^{6}}, \frac{\left|t^{2}-3 t+1\right|}{t^{7}+t^{4}+1}\right\} \\
& \quad \leq \max \left\{1, \frac{3 M^{2}}{\left(1+N^{3}\right)^{2}}\right\} \sum_{s=n}^{\infty} \sum_{t=s}^{\infty} \frac{s}{t^{4}} \\
& \quad \leq \max \left\{1, \frac{3 M^{2}}{\left(1+N^{3}\right)^{2}}\right\} \sum_{s=n}^{\infty} \sum_{t=s}^{\infty} \frac{1}{t^{3}} \\
& \quad=\max \left\{1, \frac{3 M^{2}}{\left(1+N^{3}\right)^{2}}\right\} \sum_{t=n}^{\infty} \frac{t-n+1}{t^{3}} \\
& \quad \leq \max \left\{1, \frac{3 M^{2}}{\left(1+N^{3}\right)^{2}}\right\} \sum_{t=n}^{\infty} \frac{1}{t^{2}}<+\infty, \quad \forall n \in \mathbb{N}_{n_{0}},
\end{aligned}
$$

which together with Lemma 2 yield that (13) and (14) hold. It follows from Theorem 3 that (52) possesses uncountably many unbounded positive solutions in $A(N, M)$. On the other hand, for any $L \in(N, M)$, there exist $\theta \in$ $(0,1)$ and $T \geq n_{0}+\tau+\beta$ such that for each $x_{0}=$ $\left\{x_{0 n}\right\}_{n \in \mathbb{N}_{\beta}} \in A(N, M)$, the Mann iterative sequence with errors $\left\{x_{m}\right\}_{m \in \mathbb{N}_{0}}=\left\{x_{m n}\right\}_{(m, n) \in \mathbb{N}_{0} \times \mathbb{N}_{\beta}}$ generated by (16) converges to an unbounded positive solution $x \in A(N, M)$ of (52) and has the error estimate (17), where $\left\{\gamma_{m}\right\}_{m \in \mathbb{N}_{0}}=$ $\left\{\gamma_{m n}\right\}_{(m, n) \in \mathbb{N}_{0} \times \mathbb{N}_{\beta}}$ is an arbitrary sequence in $A(N, M)$ and $\left\{\alpha_{m}\right\}_{m \in \mathbb{N}_{0}}$ and $\left\{\beta_{m}\right\}_{m \in \mathbb{N}_{0}}$ are any sequences in $[0,1]$ satisfying (18).

Example 9. Consider the second-order nonlinear neutral delay difference equation:

$$
\begin{aligned}
\Delta^{2}\left(x_{n}+x_{n-\tau}\right)+\Delta\left(\frac{\sin ^{2} x_{3 n^{3}+1}}{n^{3}\left(n^{2}+2\right)\left(1+x_{2 n^{2}-3}^{4}\right)}\right) \\
\quad+\frac{(-1)^{n} n^{3}\left(x_{n^{2}-n-1}+x_{(n+1)(n+2)}\right)}{\left(n^{11}+n^{5}+1\right)\left(1+x_{n^{2}-n-1}^{2}+x_{(n+1)(n+2)}^{2}\right)} \\
=\frac{n^{2}-\ln n}{n^{6}+n^{5}+1}, \quad n \geq 5,
\end{aligned}
$$

where $\tau \in \mathbb{N}$ is fixed. Let $n_{0}=5, k=2, \beta=5-\tau, M$ and $N$ two positive constants with $M>N$ and

$$
\begin{gathered}
a_{n}=1, \quad b_{n}=\frac{n^{2}-\ln n}{n^{6}+n^{5}+1}, \\
f(n, u, v)=\frac{(-1)^{n} n^{3}(u+v)}{\left(n^{11}+n^{5}+1\right)\left(1+u^{2}+v^{2}\right)}, \\
h(n, u, v)=\frac{\sin ^{2} v}{n^{3}\left(n^{2}+2\right)\left(1+u^{4}\right)}, \\
f_{1 n}=n^{2}-n-1, \quad F_{n}=f_{2 n}=(n+1)(n+2), \\
h_{1 n}=2 n^{2}-3, \quad H_{n}=h_{2 n}=3 n^{3}+1, \\
P_{n}=Q_{n}=\frac{4}{n^{8}}, \quad R_{n}=W_{n}=\frac{10}{n^{5}}, \quad \forall(n, u, v) \in \mathbb{N}_{n_{0}} \times \mathbb{R}^{2} .
\end{gathered}
$$


It is clear that (11), (12), and (35) are fulfilled. Note that

$$
\begin{aligned}
& \lim _{n \rightarrow \infty} \frac{1}{n} \sum_{s=n}^{\infty} \max \left\{R_{s} H_{s}, W_{s}\right\} \\
&=\lim _{n \rightarrow \infty} \frac{1}{n} \sum_{s=n}^{\infty} \max \left\{\frac{10\left(3 s^{3}+1\right)}{s^{5}}, \frac{10}{s^{5}}\right\}=0, \\
& \frac{1}{n} \sum_{s=n}^{\infty} \sum_{t=s}^{\infty} \max \left\{P_{t} F_{t}, Q_{t},\left|b_{t}\right|\right\} \\
& \quad=\frac{1}{n} \sum_{s=n}^{\infty} \sum_{t=s}^{\infty} \max \left\{\frac{4(t+1)(t+2)}{t^{8}}, \frac{4}{t^{8}}, \frac{t^{2}-\ln t}{t^{6}+t^{5}+1}\right\} \\
& \leq \frac{4}{n} \sum_{s=n}^{\infty} \sum_{t=s}^{\infty} \frac{1}{t^{4}} \leq \frac{4}{n} \sum_{t=n}^{\infty} \frac{1}{t^{3}} \longrightarrow 0 \text { as } n \longrightarrow \infty
\end{aligned}
$$

which yields that

$$
\lim _{n \rightarrow \infty} \frac{1}{n} \sum_{s=n}^{\infty} \sum_{t=s}^{\infty} \max \left\{P_{t} F_{t}, Q_{t},\left|b_{t}\right|\right\}=0
$$

Thus, Theorem 4 guarantees that (56) possesses uncountably unbounded positive solutions in $A(N, M)$. On the other hand, for any $L \in(N, M)$, there exist $\theta \in(0,1)$ and $T \geq \tau+n_{0}+\beta$ such that the Mann iterative sequence with error $\left\{x_{m}\right\}_{m \in \mathbb{N}_{0}}$ generated by (36) converges to an unbounded positive solution $x \in A(N, M)$ of (56) and has the error estimate (17), where $\left\{\gamma_{m}\right\}_{m \in \mathbb{N}_{0}}$ is an arbitrary sequence in $A(N, M)$ and $\left\{\alpha_{m}\right\}_{m \in \mathbb{N}_{0}}$ and $\left\{\beta_{m}\right\}_{m \in \mathbb{N}_{0}}$ are any sequences in $[0,1]$ satisfying $(18)$.

Example 10. Consider the second-order nonlinear neutral delay difference equation:

$$
\begin{aligned}
\Delta^{2}\left(x_{n}\right. & \left.+\frac{3 n^{3}-1}{4 n^{3}+2} x_{n-\tau}\right) \\
& +\Delta\left(\frac{\sin \left(\ln \left(1+n^{2}\left|x_{3 n^{2}-1}\right|\right)\right)}{n^{9}-\sqrt{n}-4}\right. \\
& \left.-\frac{n^{2}-(-1)^{n(n-1) / 2}}{\left(n^{7}+3 n^{5}-1\right) 2^{\left|x_{4 n^{3}+1}\right|}}\right) \\
& +\frac{(-1)^{n}}{n^{6}\left(1+x_{n-2}^{2}\right)}-\frac{1}{\left(n^{5}+1\right) \sqrt{1+\left|x_{n+4}\right|}} \\
= & \frac{(-1)^{n} n^{3}-1}{n^{8} \ln ^{3} n+1}, \quad n \geq 7,
\end{aligned}
$$

where $\tau \in \mathbb{N}$ is fixed. Let $n_{0}=7, k=2, a=3 / 4, \beta=\min \{7-$ $\tau, 5\}, M$ and $N$ two positive constants with $M>4 N$ and

$$
\begin{gathered}
a_{n}=\frac{3 n^{3}-1}{4 n^{3}+2}, \quad b_{n}=\frac{(-1)^{n} n^{3}-1}{n^{8} \ln ^{3} n+1}, \\
f(n, u, v)=\frac{(-1)^{n}}{n^{6}\left(1+u^{2}\right)}-\frac{1}{\left(n^{5}+1\right) \sqrt{1+|v|}}, \\
h(n, u, v)=\frac{\sin \left(\ln \left(1+n^{2}|u|\right)\right)}{n^{9}-\sqrt{n}-4}-\frac{n^{2}-(-1)^{n(n-1) / 2}}{\left(n^{7}+3 n^{5}-1\right) 2^{|v|}}, \\
f_{1 n}=n-2, \\
P_{n}=f_{2 n}=n+4, \quad h_{1 n}=3 n^{2}-1, \\
H_{n}=h_{2 n}=4 n^{3}+1, \\
R_{n}=W_{n}=\frac{2}{n^{5}}, \quad \forall(n, u, v) \in \mathbb{N}_{n_{0}} \times \mathbb{R}^{2} .
\end{gathered}
$$

It is not difficult to verify that (11), (12), and (42) are fulfilled. Note that

$$
\begin{aligned}
\lim _{n \rightarrow \infty} & \frac{1}{n} \sum_{s=n}^{\infty} \max \left\{R_{s} H_{s}, W_{s}\right\} \\
& =\lim _{n \rightarrow \infty} \frac{1}{n} \sum_{s=n}^{\infty} \max \left\{\frac{2\left(4 s^{3}+1\right)}{s^{5}}, \frac{2}{s^{5}}\right\} \\
& =\lim _{n \rightarrow \infty} \frac{1}{n} \sum_{s=n}^{\infty} \frac{2\left(4 s^{3}+1\right)}{s^{5}}=0, \\
\lim _{n \rightarrow \infty} & \frac{1}{n} \sum_{s=n}^{\infty} \sum_{t=s}^{\infty} \max \left\{P_{t} F_{t}, Q_{t},\left|b_{t}\right|\right\} \\
& =\lim _{n \rightarrow \infty} \frac{1}{n} \sum_{s=n}^{\infty} \sum_{t=s}^{\infty} \max \left\{\frac{2 t+8}{t^{4}}, \frac{2}{t^{4}}, \frac{\left|(-1)^{t} t^{3}-1\right|}{t^{8} \ln ^{3} t+1}\right\} \\
& =\lim _{n \rightarrow \infty} \frac{1}{n} \sum_{s=n}^{\infty} \sum_{t=s}^{\infty} \frac{2 t+8}{t^{4}}=0 .
\end{aligned}
$$

That is, (33) and (34) are satisfied. Consequently Theorem 5 implies that (60) possesses uncountably many unbounded positive solutions in $A(N, M)$. On the other hand, for any $L \in((3 / 4) M+N, M)$, there exist $\theta \in(0,1)$ and $T \geq$ $n_{0}+\tau+\beta$ such that the Mann iterative sequence with error $\left\{x_{m}\right\}_{m \in \mathbb{N}_{0}}$ generated by (43) converges to an unbounded positive solution $x \in A(N, M)$ of (60) and has the error estimate (17), where $\left\{\gamma_{m}\right\}_{m \in \mathbb{N}_{0}}$ is an arbitrary sequence in $A(N, M)$ and $\left\{\alpha_{m}\right\}_{m \in \mathbb{N}_{0}}$ and $\left\{\beta_{m}\right\}_{m \in \mathbb{N}_{0}}$ are any sequences in $[0,1]$ satisfying $(18)$. 
Example 11. Consider the second-order nonlinear neutral delay difference equation:

$$
\begin{aligned}
\Delta^{2}\left(x_{n}\right. & \left.+\frac{1-2 n^{2}}{2+3 n^{2}} x_{n-\tau}\right) \\
& +\Delta\left(\frac{n^{2}-1}{\left(n^{5}+2 n^{3}-1\right)\left(1+x_{2 n-15}^{2}\right)}\right) \\
& +\frac{\sin ^{2}\left(n^{3} x_{5 n^{2}-2}\right)}{(n+2)^{8}} \\
= & \frac{(-1)^{n} n^{4}+n^{3}+3 n^{2}-1}{n^{9}+n^{7}+3 n^{6}+n^{4}+1}, \quad n \geq 11,
\end{aligned}
$$

where $\tau \in \mathbb{N}$ is fixed. Let $n_{0}=11, k=1, a=-4 / 5, \beta=$ $\min \{11-\tau, 7\}, M$ and $N$ two positive constants with $M>5 N$ and

$$
\begin{gathered}
a_{n}=\frac{1-2 n^{2}}{2+3 n^{2}}, \quad b_{n}=\frac{(-1)^{n} n^{4}+n^{3}+3 n^{2}-1}{n^{9}+n^{7}+3 n^{6}+n^{4}+1}, \\
f(n, u)=\frac{\sin ^{2}\left(n^{3} u\right)}{(n+2)^{8}}, \\
h(n, u)=\frac{n^{2}-1}{\left(n^{5}+2 n^{3}-1\right)\left(1+u^{2}\right)}, \\
H_{n}=h_{1 n}=2 n-15, \quad f_{1 n}=5 n^{2}-2, \\
R_{n}=\frac{2}{n^{3}}, \quad W_{n}=\frac{1}{n^{3}}, \quad \forall(n, u) \in \mathbb{N}_{n_{0}} \times \mathbb{R} .
\end{gathered}
$$

Obviously, (11), (12), and (50) are satisfied. Note that

$$
\begin{aligned}
& \lim _{n \rightarrow \infty} \frac{1}{n} \sum_{s=n}^{\infty} \max \left\{R_{s} H_{s}, W_{s}\right\} \\
& =\lim _{n \rightarrow \infty} \frac{1}{n} \sum_{s=n}^{\infty} \max \left\{\frac{4 s-30}{s^{3}}, \frac{1}{s^{3}}\right\}=0, \\
& \frac{1}{n} \sum_{s=n}^{\infty} \sum_{t=s}^{\infty} \max \left\{P_{t} F_{t}, Q_{t},\left|b_{t}\right|\right\} \\
& =\frac{1}{n} \sum_{s=n}^{\infty} \sum_{t=s}^{\infty} \max \left\{\frac{2\left(5 t^{2}-2\right)}{t^{5}}, \frac{1}{t^{8}}, \frac{\left|(-1)^{t} t^{4}+t^{3}+3 t^{2}-1\right|}{t^{9}+t^{7}+3 t^{6}+t^{4}+1}\right\} \\
& \leq \frac{10}{n} \sum_{s=n}^{\infty} \sum_{t=s}^{\infty} \frac{1}{t^{3}} \longrightarrow 0 \quad \text { as } n \longrightarrow \infty,
\end{aligned}
$$

which gives that

$$
\lim _{n \rightarrow \infty} \frac{1}{n} \sum_{s=n}^{\infty} \sum_{t=s}^{\infty} \max \left\{P_{t} F_{t}, Q_{t},\left|b_{t}\right|\right\}=0
$$

That is, (33) and (34) hold. Thus, Theorem 6 shows that (63) possesses uncountably many unbounded positive solutions in $A(N, M)$. On the other hand, for any $L \in(N, M / 5)$, there exist $\theta \in(0,1)$ and $T \geq n_{0}+\tau+\beta$ such that the Mann iterative sequence with error $\left\{x_{m}\right\}_{m \in \mathbb{N}_{0}}$ generated by (43) converges to an unbounded positive solution $x \in A(N, M)$ of (63) and has the error estimate (17), where $\left\{\gamma_{m}\right\}_{m \in \mathbb{N}_{0}}$ is an arbitrary sequence in $A(N, M)$ and $\left\{\alpha_{m}\right\}_{m \in \mathbb{N}_{0}}$ and $\left\{\beta_{m}\right\}_{m \in \mathbb{N}_{0}}$ are any sequences in $[0,1]$ satisfying (18).

\section{Acknowledgments}

This research was supported by the Science Research Foundation of Educational Department of Liaoning Province (L2012380) and Basic Science Research Program through the National Research Foundation of Korea (NRF) funded by the Ministry of Education, Science and Technology (2012R1A1A2002165).

\section{References}

[1] M. H. Abu-Risha, "Oscillation of second-order linear difference equations," Applied Mathematics Letters, vol. 13, no. 1, pp. 129$135,2000$.

[2] R. P. Agarwal, Difference Equations and Inequalities, vol. 228 of Monographs and Textbooks in Pure and Applied Mathematics, Marcel Dekker, New York, NY, USA, 2nd edition, 2000.

[3] R. P. Agarwal, M. M. S. Manuel, and E. Thandapani, "Oscillatory and nonoscillatory behavior of second-order neutral delay difference equations. II," Applied Mathematics Letters, vol. 10, no. 2, pp. 103-109, 1997.

[4] S. S. Cheng, H. J. Li, and W. T. Patula, "Bounded and zero convergent solutions of second-order difference equations," Journal of Mathematical Analysis and Applications, vol. 141, no. 2, pp. 463-483, 1989.

[5] C. Jinfa, "Existence of a nonoscillatory solution of a secondorder linear neutral difference equation," Applied Mathematics Letters, vol. 20, no. 8, pp. 892-899, 2007.

[6] W. T. Li and S. S. Cheng, "Oscillation criteria for a nonlinear difference equation," Computers \& Mathematics with Applications, vol. 36, no. 8, pp. 87-94, 1998.

[7] W.-T. Li and S.-S. Cheng, "Remarks on two recent oscillation theorems for second-order linear difference equations," Applied Mathematics Letters, vol. 16, no. 2, pp. 161-163, 2003.

[8] W.-T. Li and S. H. Saker, "Oscillation of second-order sublinear neutral delay difference equations," Applied Mathematics and Computation, vol. 146, no. 2-3, pp. 543-551, 2003.

[9] X. Li and D. Zhu, "New results for the asymptotic behavior of a nonlinear second-order difference equation," Applied Mathematics Letters, vol. 16, no. 5, pp. 627-633, 2003.

[10] H. Liang and P. Weng, "Existence and multiple solutions for a second-order difference boundary value problem via critical point theory," Journal of Mathematical Analysis and Applications, vol. 326, no. 1, pp. 511-520, 2007.

[11] Z. Liu, S. M. Kang, and J. S. Ume, "Existence of uncountably many bounded nonoscillatory solutions and their iterative approximations for second order nonlinear neutral delay difference equations," Applied Mathematics and Computation, vol. 213, no. 2, pp. 554-576, 2009. 
[12] Z. Liu, Y. Xu, and S. M. Kang, "Global solvability for a second order nonlinear neutral delay difference equation," Computers \& Mathematics with Applications, vol. 57, no. 4, pp. 587-595, 2009.

[13] J. W. Luo and D. D. Bainov, "Oscillatory and asymptotic behavior of second-order neutral difference equations with maxima," Journal of Computational and Applied Mathematics, vol. 131, no. 1-2, pp. 333-341, 2001.

[14] M. Ma, H. Tang, and W. Luo, "Periodic solutions for nonlinear second-order difference equations," Applied Mathematics and Computation, vol. 184, no. 2, pp. 685-694, 2007.

[15] Q. Meng and J. Yan, "Bounded oscillation for second-order nonlinear delay differential equation in a critical state," Journal of Computational and Applied Mathematics, vol. 197, no. 1, pp. 204-211, 2006.

[16] M. Migda and J. Migda, "Asymptotic properties of solutions of second-order neutral difference equations," Nonlinear Analysis, Theory, Methods and Applications, vol. 63, no. 5-7, pp. e789e799, 2005.

[17] S. H. Saker, "New oscillation criteria for second-order nonlinear neutral delay difference equations," Applied Mathematics and Computation, vol. 142, no. 1, pp. 99-111, 2003.

[18] M.-C. Tan and E.-H. Yang, "Oscillation and nonoscillation theorems for second order nonlinear difference equations," Journal of Mathematical Analysis and Applications, vol. 276, no. 1, pp. 239-247, 2002.

[19] X. H. Tang, "Bounded oscillation of second-order delay difference equations of unstable type," Computers \& Mathematics with Applications, vol. 44, no. 8-9, pp. 1147-1156, 2002.

[20] E. Thandapani, M. M. S. Manuel, J. R. Graef, and P. W. Spikes, "Monotone properties of certain classes of solutions of secondorder difference equations," Computers \& Mathematics with Applications, vol. 36, no. 10-12, pp. 291-297, 1998.

[21] Y. Tian and W. Ge, "Multiple positive solutions of boundary value problems for second-order discrete equations on the halfline," Journal of Difference Equations and Applications, vol. 12, no. 2, pp. 191-208, 2006.

[22] B. G. Zhang, "Oscillation and asymptotic behavior of second order difference equations," Journal of Mathematical Analysis and Applications, vol. 173, no. 1, pp. 58-68, 1993.

[23] Z. Zhang, J. Chen, and C. Zhang, "Oscillation of solutions for second-order nonlinear difference equations with nonlinear neutral term," Computers \& Mathematics with Applications, vol. 41, no. 12, pp. 1487-1494, 2001.

[24] Z. Zhang and Q. Li, "Oscillation theorems for second-order advanced functional difference equations," Computers \& Mathematics with Applications, vol. 36, no. 6, pp. 11-18, 1998.

[25] Z. G. Zhang and J. S. Yu, "On existence of positive solutions for neutral differential equations," Science in China. Series A, vol. 8, pp. 785-790, 1992.

[26] Z. Zhang and J. Zhang, "Oscillation criteria for second-order functional difference equations with "summation small" coefficient," Computers \& Mathematics with Applications, vol. 38, no. 1, pp. 25-31, 1999.

[27] L. S. Liu, "Ishikawa and Mann iterative process with errors for nonlinear strongly accretive mappings in Banach spaces," Journal of Mathematical Analysis and Applications, vol. 194, no. 1, pp. 114-125, 1995. 


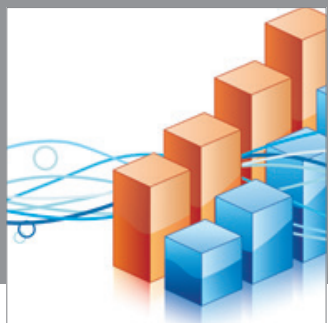

Advances in

Operations Research

mansans

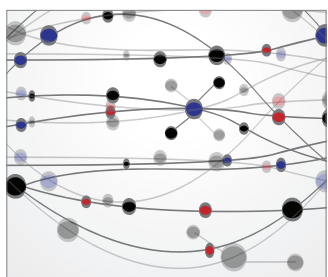

The Scientific World Journal
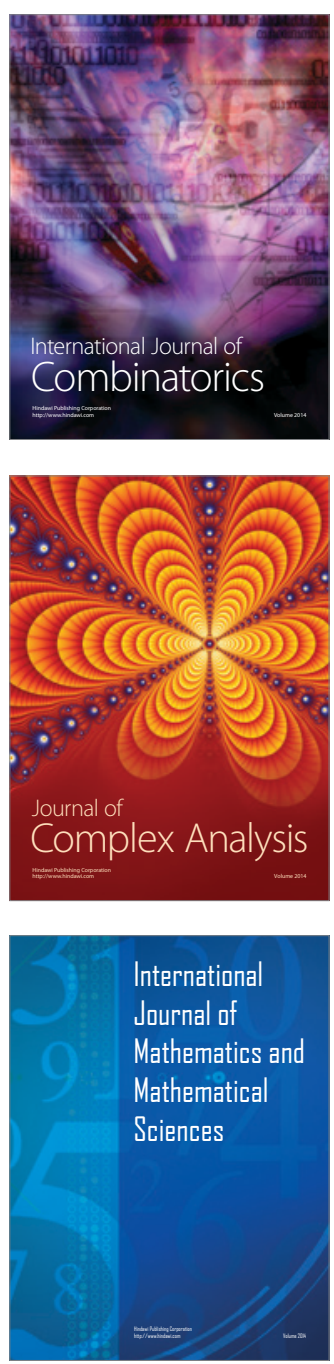
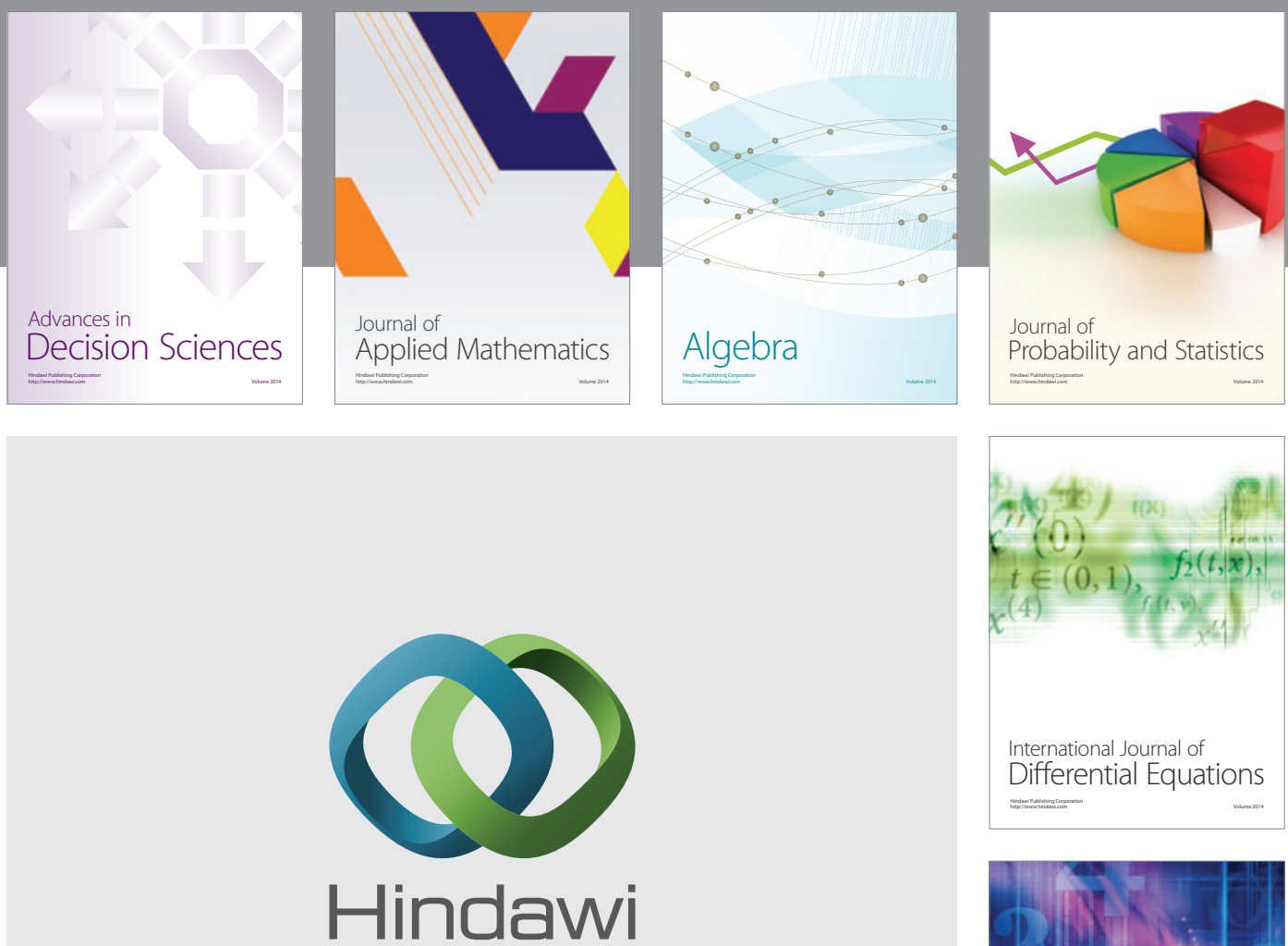

Submit your manuscripts at http://www.hindawi.com
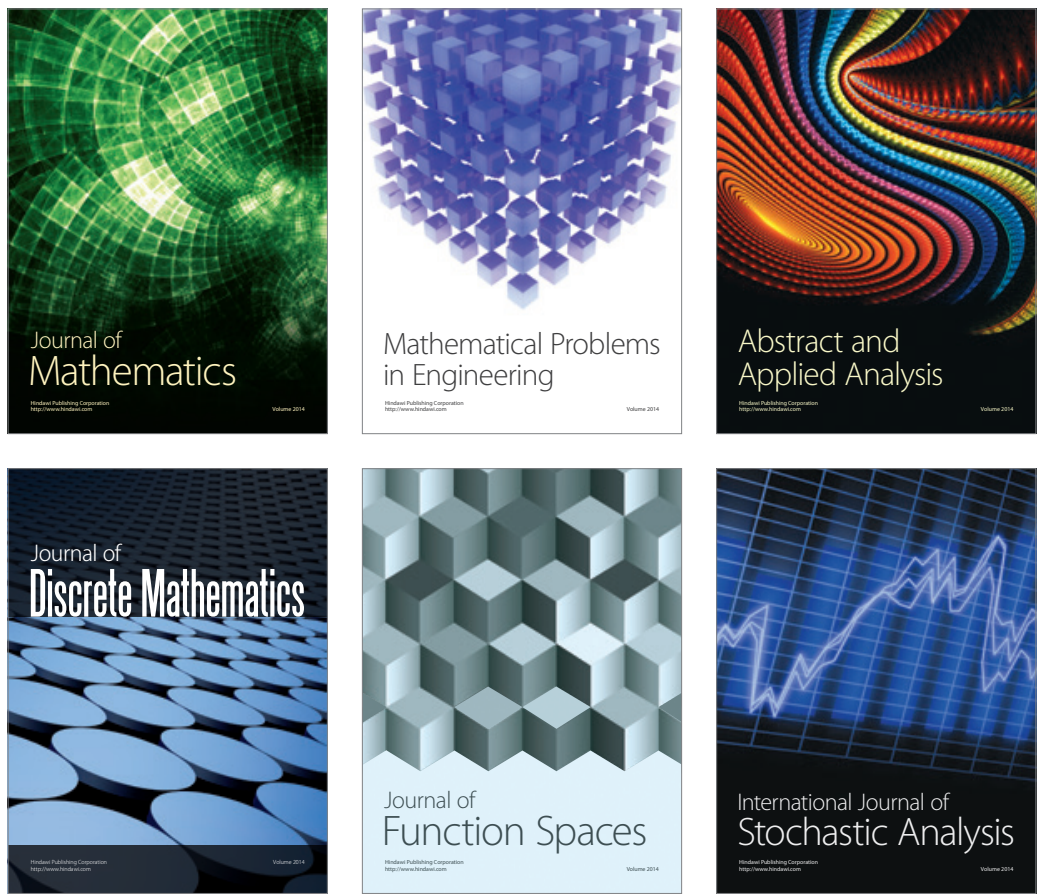

Journal of

Function Spaces

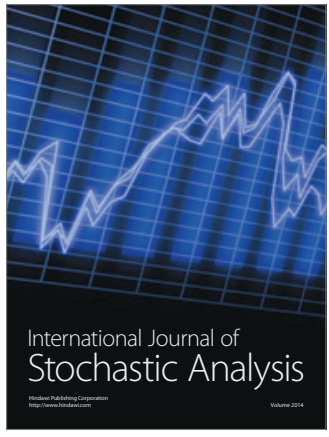

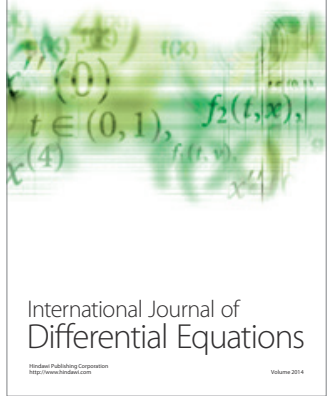
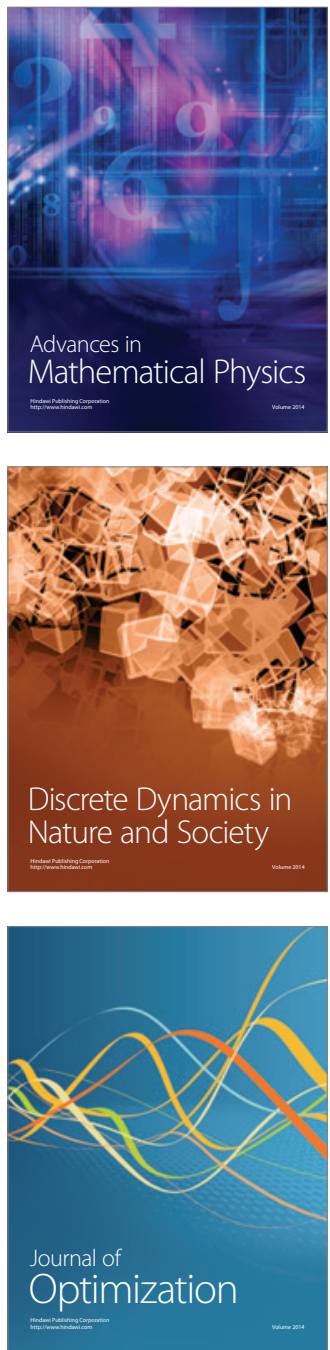